\title{
TEORES DE METAIS PESADOS, K E Na, NO SUBSTRATO, EM FUNÇÃO DE DOSES DE COMPOSTO ORGÂNICO DE LIXO URBANO E DE CULTIVARES DE ALFACE
}

\author{
HEAVY METALS, K AND Na CONTENTS IN SUBSTRATE IN FUNCTION OF DOSES \\ OF ORGANIC COMPOST OF URBAN WASTE AND LETTUCE CULTIVARS
}

\author{
Izabel Cristina dos Santos ${ }^{1}$ Vicente Wagner Dias Casali ${ }^{2}$ Glauco Vieira Miranda ${ }^{3}$
}

RESUMO

Visando a avaliar os teores de metais pesados, $K e$ Na, no substrato, após a colheita da alface, foi instalado um ensaio com os cultivares Brasil 48, Regina 71, Vitória Verde Clara e Grand Rapids e as doses de composto orgânico de lixo urbano $0,17,5,35$ e 52,5t ha ${ }^{-1}$, em casa de vegetação. Foram analisados: $\mathrm{pH}$ em água, condutividade elétrica, carbono orgânico e teores disponíveis de $\mathrm{Pb}, \mathrm{Cu}, \mathrm{Mn}, \mathrm{Zn}, \mathrm{K}$ e $\mathrm{Na}$ no solo. $\mathrm{Os}$ resultados demonstraram que as doses de composto orgânico de lixo urbano alteraram os valores de carbono orgânico, condutividade elétrica, $\mathrm{pH}$ e os teores disponíveis de $\mathrm{Pb}, \mathrm{Zn}, \mathrm{Cu}, \mathrm{Mn}, \mathrm{K}$ e $\mathrm{Na}$ do substrato após o cultivo dos quatro cultivares. Estes influenciaram os valores de carbono orgânico e os teores de Zn, $\mathrm{Cu}, \mathrm{Mn}, \mathrm{K}$ e Na do mesmo substrato. Quanto maior as doses utilizadas de composto de lixo urbano, maior o teor de Cu, Mn $\mathrm{Pb}, \mathrm{Zn}, \mathrm{K}$, Na e maiores o carbono orgânico, a condutividade elétrica e o pH do substrato após o cultivo da alface.

Palavras-chave: metais pesados, composto orgânico, lixo urbano, alface.

\section{SUMMARY}

The objective of this experiment was to evaluated the heavy metal, Kand Na in the substrate after the harvest of lettuce, in a experiment with cultivars Brasil 48, Regina 71, Vitória Verde Clara e Grand Rapids and doses of organic compost of municipal waste $0,17.5,35$ e $52.5 \mathrm{tha}^{-1}$, in greenhouse. The variables analised were $\mathrm{pH}$ in water, electric conductivit, organic carbon, and avaiable contents of $\mathrm{Pb}, \mathrm{Cu}, \mathrm{Mn}, \mathrm{Zn}, \mathrm{K}$ and $\mathrm{Na}$ in the soil. Results demonstrated that doses of organic compost disturbed the values of $\mathrm{pH}$, organic carbon, electric conductivit, and the avaiable contents of $\mathrm{Pb}, \mathrm{Cu}, \mathrm{Mn}, \mathrm{Zn}, \mathrm{K}$ and $\mathrm{Na}$ in the substrate after the harvest of the four cultivars. Lettuce cultivars influenced the values of organic carbon and the contents of $\mathrm{Cu}, \mathrm{Mn}, \mathrm{Zn} \mathrm{K}$ and $\mathrm{Na}$ of the same substrate. Increasing the dose of organic compost, values of $\mathrm{Pb}, \mathrm{Cu}, \mathrm{Mn}, \mathrm{Zn}, \mathrm{K}, \mathrm{Na}, \mathrm{pH}$, electric condutivit and organic carbon were increased.

Key words: heavy metals, organic compost, urban waste, lettuce.

\section{INTRODUÇÃO}

A utilização de adubo orgânico tem sido uma boa opção para reduzir os gastos com fertilizantes minerais, e obter aumento de produtividade no cultivo de alface. No entanto, há estudos comprovando que a partir de determinados níveis, o adubo orgânico pode limitar a produção por provocar salinização do solo, devido à elevada concentração de íons, os quais variam de acordo com o material que deu origem ao adubo orgânico (CHANYASAK et al., 1983; COSTA, 1994).

A utilização do composto orgânico de lixo urbano na agricultura brasileira tem se difundido nos últimos anos, especialmente próximo às usinas de reciclagem, mas ainda são escassas informações consistentes quanto à sua composição química e quanto à dose adequada para as diferentes espécies. KIEHL (1985) afirma que a composição química e física destes resíduos é extremamente variável com a classe social e a época do ano. Os resultados apresentados por KAUSS (1989) revelaram a existência de altos índices de contaminação com metais pesados em composto orgânico de quatros usinas de lixo brasileiras, comparadas ao padrão alemão.

\footnotetext{
${ }^{1}$ Engenheiro Agrônomo, MSc., Departamento de Fitotecnia. Universidade Federal de Viçosa (UFV). Bolsista do CNPq

${ }^{2}$ Engenheiro Agrônomo, PhD., Professor Titular,. Departamento de Fitotecnia, UFV.

${ }^{3}$ Engenheiro Agrônomo, Professor Adjunto, Doutor, Departamento de Fitotecnia, UFV, 36571-000, Viçosa, MG. Autor para correspondência.
} 
A mobilidade e retenção de metais pesados no solo dependem das complexas interações com a fase sólida, tanto orgânica quanto inorgânica (BUCKMAN \& BRADY, 1979), que envolvem reações de adsorção/dessorção, precipitação/dissolução, complexação e oxirredução (AMARAL SOBRINHO, 1992). Os metais pesados mais perigosos pela toxicidade e potencial de bioacumulação são: $\mathrm{Cd}, \mathrm{Cu}, \mathrm{Zn}$ e $\mathrm{Pb}$ (MAEDA $\boldsymbol{e t}$ al., 1990; CHANG et al., 1984).

Vários autores afirmam que espécies vegetais e até cultivares diferem quanto à capacidade de absorção e acúmulo de metais pesados (ZURERA et al., 1987; NICKLOW et $\boldsymbol{a l} ., 1983)$. A alface é considerada uma das espécies mais eficientes na absorção de tais elementos (NICKLOW et $\boldsymbol{a l}$., 1983). Como cultivares de alface apresentam comportamento diferencial quanto à quantidade e velocidade de absorção de macro e micronutrientes (RODRIGUES, 1994), supôs-se que também apresentariam tal comportamento em relação a outros elementos, incluindo metais pesados.

Este trabalho teve por objetivo verificar o efeito da adubação com composto orgânico de lixo urbano sobre $\mathrm{pH}$ em água, condutividade eléctrica, carbono orgânico, teores de $\mathrm{Pb}, \mathrm{Cu}, \mathrm{Mn}, \mathrm{Zn}, \mathrm{K}$ e Na no substrato, após o cultivo de alface.

\section{MATERIAL E MÉTODOS}

Em 1993 foi conduzido um experimento em casa de vegetação, na Universidade Federal de Viçosa (UFV), Viçosa, MG, que consistiu no transplante de mudas de alface (Lactuca sativa L.) com 21 dias, em vasos de plástico com capacidade para $5 \mathrm{dm}^{3}$. O experimento fatorial consistiu na combinação de quatro cultivares de alface (Brasil 48, Grand Rapids, Vitória Verde Clara e Regina 71) e quatro doses de composto orgânico de lixo urbano, dispostos no delineamento em blocos ao acaso, com quatro repetições. As doses testadas, em base seca, foram: $0 \mathrm{t} \mathrm{ha}{ }^{-1}$ (testemunha); 17,5 $\mathrm{ha}^{-1}(1 / 2$ da dose recomendada pela COMISSÃO... (1989) para a alface); $35 \mathrm{t} \mathrm{ha}^{-1}$ (dose recomendada) e 52,5t ha ${ }^{-1}(1,5$ vezes a dose recomendada). A sementeira foi preparada utilizando-se substrato não contaminado (verificado por meio de análise química) e recebeu uma irrigação com solução nutritiva completa (MARTINEZ, 1988), com $25 \%$ da concentração inicial e irrigações diárias com água desmineralizada.

Para preparo do substrato dos vasos, utilizou-se composto orgânico de lixo urbano, cedido pela Companhia de Limpeza Urbana do Rio de Janeiro (COMLURB, RJ), oriundo da Usina de Jacarepaguá, retirado de material disponível para venda e uso pelos agricultores, e material de um Latossolo vermelho-amarelo, textura argilosa, proveniente do Município de Machado-MG, fornecido pelo Banco de Solos do Departamento de Solos da UFV. O pH do material de solo foi corrigido por meio da aplicação de carbonatos de cálcio e magnésio p.a.. As características físicas e químicas do composto orgânico de lixo urbano, após seco ao ar, encontram-se na tabela 1. As características do solo e dos substratos antes de receberem adubação mineral são apresentadas na tabela 2 .

Após incubação por 26 dias com irrigação periódica com água desmineralizada, o substrato recebeu adubação mineral. Cada vaso de $5 \mathrm{dm}^{3}$ recebeu $150 \mathrm{ml}$ de solução nutritiva que foram manualmente homogeneizados, de modo que cada $\mathrm{dm}^{3}$ de substrato recebeu: $50 \mathrm{mg}$ de nitrogênio, $100 \mathrm{mg}$ de fósforo e $50 \mathrm{mg}$ de potássio, na forma dos seguintes sais: $\mathrm{NH}_{4} \mathrm{NO}_{3}, \mathrm{NH}_{4} \mathrm{H}_{2} \mathrm{PO}_{4}$ e $\mathrm{KH}_{2} \mathrm{PO}_{4}$. A aduba-

Tabela 1 - Características químicas e físicas do composto orgânico de lixo urbano, na época da incorporação ao solo, UFV, Viçosa, MG. 1993.

\begin{tabular}{|c|c|}
\hline Características avaliadas & Valor \\
\hline pH em água $(1: 2,5)^{1 /}$ & 7,80 \\
\hline Matéria orgânica total $(\%)^{1 /}$ & 41,91 \\
\hline Carbono total $(\%)^{1 /}$ & 24,31 \\
\hline Carbono Orgânico $(\%)^{1 /}$ & 21,00 \\
\hline $\mathrm{N}$ - Total $(\%)^{2 /}$ & 1,21 \\
\hline $\mathrm{P}-\mathrm{Total}(\%)^{2 /}$ & 0,40 \\
\hline $\mathrm{K}$ - Total $(\%)^{2 /}$ & 0,70 \\
\hline $\mathrm{Ca}-\mathrm{Total}(\%)^{2 /}$ & 4,10 \\
\hline $\operatorname{Mg}$ - Total $(\%)^{2 /}$ & 0,20 \\
\hline S - Total $(\%)^{2 /}$ & 0,27 \\
\hline $\mathrm{Na}\left(\mu \mathrm{g} \cdot \mathrm{g}^{-1}\right)^{1 /}$ & 1960,80 \\
\hline $\mathrm{B}\left(\mu \mathrm{g} \cdot \mathrm{g}^{-1}\right)^{2 /}$ & 29,00 \\
\hline $\mathrm{Pb}\left(\mu \mathrm{g} . \mathrm{g}^{-1}\right)^{2 /}$ & 214,50 \\
\hline $\mathrm{Zn}\left(\mu \mathrm{g} \cdot \mathrm{g}^{-1}\right)^{2 /}$ & 363,00 \\
\hline $\mathrm{Cu}\left(\mu \mathrm{g} \cdot \mathrm{g}^{-1}\right)^{2 /}$ & 204,00 \\
\hline $\operatorname{Mn}\left(\mu \mathrm{g} \cdot \mathrm{g}^{-1}\right)^{2 /}$ & 175,00 \\
\hline $\mathrm{Cd}\left(\mu \mathrm{g} \cdot \mathrm{g}^{-1}\right)^{3 /}$ & 1,24 \\
\hline $\mathrm{Ni}\left(\mu \mathrm{g} \cdot \mathrm{g}^{-1}\right)^{2 /}$ & 28,20 \\
\hline Relação C/N & 17,35 \\
\hline Densidade $\left(\mathrm{g} / \mathrm{cm}^{2}\right)$ & 0,50 \\
\hline Umidade $(\%)^{1 /}$ & 21,00 \\
\hline Granulometria $(3-2 \mathrm{~mm})^{2 /}$ & 4,53 \\
\hline$(\%) \quad(3-1 \mathrm{~mm})^{2 /}$ & 40,03 \\
\hline$(<1 \mathrm{~mm})^{2 /}$ & 45,04 \\
\hline
\end{tabular}

${ }^{1 /}$ Resultados extraídos da análise realizada pelo Laboratório de Análises de Rotina do Departamento de Solos da UFV.

${ }^{2 /}$ Resultados extraídos da análise realizada pelo Laboratório de Solos da ARACRUZ CELULOSE S.A.

${ }^{3 /}$ Resultados extraídos da análise realizada no Laboratório de Nutrição Mineral de Plantas do Departamento de Fitotecnia da UFV. 
Tabela 2 - Características do material de solo (dose zero) e dos substratos antes do plantio, sem adubação mineral, UFV, Viçosa, MG. 1993.

\begin{tabular}{|c|c|c|c|c|}
\hline \multirow[t]{2}{*}{ Características avaliadas } & \multicolumn{4}{|c|}{ Doses de Composto $\left(\mathrm{t} \mathrm{ha}^{-1}\right)$} \\
\hline & 0 & 17,5 & 35,0 & 52,5 \\
\hline pH em água $(1: 2,5)$ & 5,4 & 6,3 & 6,8 & 7,1 \\
\hline Carbono Orgânico (\%) & 2,1 & 2,7 & 3,5 & 3,6 \\
\hline $\mathrm{P}$ disponível (mg.dm $\left.{ }^{-3}\right)$ & 4,4 & 23,9 & 39,5 & 49,2 \\
\hline K disponível (mg.dm ${ }^{-3}$ ) & 70,0 & 306,0 & 454,0 & 532,0 \\
\hline $\mathrm{Na}\left(\mathrm{mg} \cdot \mathrm{dm}^{-3}\right)$ & 15,7 & 166,7 & 245,1 & 343,1 \\
\hline Al trocável (meq. $\left.100 \mathrm{~cm}^{-3}\right)$ & 0,0 & 0,0 & 0,0 & 0,0 \\
\hline Ca trocável(meq. $\left.100 \mathrm{~cm}^{-3}\right)$ & 2,2 & 4,3 & 5,7 & 5,7 \\
\hline $\mathrm{Mg}$ trocável(meq. $\left.100 \mathrm{~cm}^{-3}\right)$ & 0,6 & 1,4 & 0,9 & 1,0 \\
\hline $\mathrm{H}+\mathrm{Al}\left(\right.$ meq. $\left.100 \mathrm{~cm}^{-3}\right)$ & 5,1 & 3,0 & 1,8 & 1,5 \\
\hline Soma de Bases (meq. $100 \mathrm{~cm}^{-3}$ ) & 2,9 & 6,5 & 7,8 & 8,0 \\
\hline CTC Total (meq. $\left.100 \mathrm{~cm}^{-3}\right)$ & 8,0 & 9,5 & 9,6 & 9,5 \\
\hline Sat.de Bases(meq. $\left.100 \mathrm{~cm}^{-3}\right)$ & 36,9 & 68,5 & 81,2 & 84,2 \\
\hline Sat.de $\mathrm{Al}\left(\right.$ meq. $\left.100 \mathrm{~cm}^{-3}\right)$ & 0,0 & 0,0 & 0,0 & 0,0 \\
\hline $\mathrm{Pb}\left(\mathrm{mg} \cdot \mathrm{dm}^{-3}\right)$ & 0,6 & 3,6 & 5,7 & 6,6 \\
\hline $\mathrm{Zn}\left(\mathrm{mg} \cdot \mathrm{dm}^{-3}\right)$ & 0,4 & 8,6 & 16,4 & 19,0 \\
\hline $\mathrm{Cu}\left(\mathrm{mg} \cdot \mathrm{dm}^{-3}\right)$ & 0,2 & 1,6 & 3,0 & 3,5 \\
\hline $\operatorname{Mn}\left(\mathrm{mg} \cdot \mathrm{dm}^{-3}\right)$ & 5,9 & 9,0 & 11,2 & 13,5 \\
\hline $\mathrm{Fe}\left(\mathrm{mg} \cdot \mathrm{dm}^{-3}\right)$ & 37,7 & 56,3 & 65,9 & 70,4 \\
\hline $\mathrm{CE}\left(\mathrm{dS} \cdot \mathrm{m}^{-1}\right)$ & 0,8 & 2,1 & $\begin{array}{r}4,9 \\
4,9\end{array}$ & $\begin{array}{r}7,4 \\
5,9\end{array}$ \\
\hline
\end{tabular}

Obs: Resultados extraídos da análise realizada pelo Laboratório de Análises de Rotina do Departamento de Solos da UFV.

ção nitrogenada em cobertura foi realizada 13 e 21 dias após o transplante, fornecendo $50 \mathrm{mg} \mathrm{N} . \mathrm{dm}^{-3}$, em cada aplicação, sendo utilizado o $\left(\mathrm{NH}_{4}\right)_{2} \mathrm{HPO}_{4}$ p.a. para evitar contaminação. A umidade dos vasos foi mantida pela irrigação com água desmineralizada, sempre que necessário, de modo a evitar o percolamento de excessos para os pratos coletores.

A colheita foi realizada 30 dias após o transplante, com o corte das plantas rente ao solo. Após separação das raízes por peneiramento, de cada unidade experimental foi retirada uma amostra do substrato para determinações do $\mathrm{pH}$ em água, pelo método potenciométrico; de $\mathrm{Pb}, \mathrm{Cu}, \mathrm{Mn}, \mathrm{Zn}, \mathrm{K}$ e Na disponíveis, pelo método do extrator duplo ácido Mehlich-1 (DEFELIPO \& RIBEIRO, 1981); do carbono orgânico (CO), em \%, pelo método Walkley e Black (DEFELIPO \& RIBEIRO, 1981) e da condutividade elétrica (CE) no extrato de saturação, conforme metodologia descrita por RHOADES (1982).

Foi realizada a análise de variância e de regressão, sendo as médias do fator qualitativo (variedades) comparadas pelo teste de Duncan, em 5\% de probabilidade. Para o fator quantitativo (doses de composto orgânico), as equações foram ajustadas de acordo com a significância dos efeitos linear, quadrático e cúbico, pelo teste de $\mathrm{F}$ em $5 \%$ de probabilidade de erro.

\section{RESULTADOS E DISCUSSÃO}

O resumo da análise de variância dos teores de $\mathrm{Pb}, \mathrm{Zn}, \mathrm{K}$, Na e dos valores de carbono orgânico (CO) encontram-se na tabela 3 e os valores do $\mathrm{pH}$ em água, da condutividade elétrica (CE) e dos teores de $\mathrm{Cu}$ e $\mathrm{Mn}$ encontram-se na tabela 4.

A análise de variância da porcentagem de $\mathrm{CO}$ do substrato mostrou efeito significativo dos cultivares e da interação cultivar $\mathrm{x}$ dose (tabela 3). O efeito das doses de composto sobre a porcentagem de $\mathrm{CO}$ foi crescente, sendo linear no substrato onde foi cultivada 'Vitória Verde Clara' e quadrático no substrato dos demais cultivares (tabela 5). As doses estimadas do teor máximo de CO foram de 56,7, 70,0 e 77,5t ha' ${ }^{-1}$ para 'Brasil 48', 'Grand Rapids' e 'Regina 71', respectivamente. HE et al. (1992) afirmam que a alteração mais significativa que o composto orgânico de lixo provoca no solo é o aumento no conteúdo de matéria orgânica, verificado neste trabalho através da porcentagem de CO $(\% \mathrm{CO}$ x 1,74=\% de matéria orgânica). DUGGAM \& WILES (1976) e GENEVINI et al. (1991) também verificaram acréscimo no conteúdo de matéria orgânica do solo com aplicação de composto orgânico de lixo urbano. HERNANDO et al. (1989) constataram, após 90 dias de incubação do solo com composto orgânico de lixo, aumento da porcentagem de agregados estáveis e da capacidade de retenção de água, ao aplicarem doses superiores a $30 \mathrm{tha}^{-1}$.

Os cultivares estudados, bem como a interação cultivares $\mathrm{x}$ doses tiveram efeito significativo sobre o teor de $\mathrm{Pb}$ do substrato após a colheita (tabela 3). O efeito das doses de composto sobre a porcentagem de $\mathrm{Pb}$ foi linear no substrato onde foi cultivado 'Vitória Verde Clara' e quadrático no substrato dos demais cultivares (tabela 5). As doses estimadas do teor máximo de $\mathrm{Pb}$ no solo foram de 70,7, 69,5 e 85t ha ${ }^{-1}$ para 'Brasil 48', 'Grand Rapids' e 'Regina 71', respectivamente. $\mathrm{O}$ teor de $\mathrm{Pb}$ do substrato, após a colheita da alface, apresentou uma grande variação, o que é compreensível, já que o teor de $\mathrm{Pb}$ no substrato aumentou de $0,6 \mathrm{mg} \cdot \mathrm{dm}^{-3}$ na

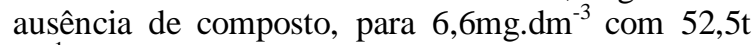
$\mathrm{ha}^{-1}$ de composto (tabela 2). No entanto, GENEVINI et al. (1991), ao utilizarem 0, 21 e 42t ha ${ }^{-1}$ de composto orgânico de lixo urbano, não encontraram teores de $\mathrm{Pb}$ significativamente diferentes entre si; provavelmente, o composto utilizado tinha baixo teor de $\mathrm{Pb}$. COSTA (1994), ao adubar alface com composto orgânico de lixo, verificou que a elevação das doses de composto $\left(0 ; 30\right.$; 60 e 90t ha $\left.{ }^{-1}\right)$ provocou aumento nos teores disponíveis de $\mathrm{Pb}$ do substrato. 
Tabela 3 - Resumo da análise de variância dos teores de $\mathrm{Pb}, \mathrm{Zn}, \mathrm{K}$, Na e dos valores de carbono orgânico (CO) dos substratos, após colheita dos cultivares de alface adubados com composto orgânico de lixo urbano.

\begin{tabular}{|c|c|c|c|c|c|c|}
\hline & \multirow[b]{2}{*}{ G.L. } & \multicolumn{5}{|c|}{ Quadrados médios } \\
\hline & & $\mathrm{CO}$ & $\mathrm{Pb}$ & $\mathrm{Zn}$ & $\mathrm{K}$ & $\mathrm{Na}$ \\
\hline Blocos & 3 & $0,01^{\mathrm{ns}}$ & $0,02^{\mathrm{ns}}$ & $0,5^{\mathrm{ns}}$ & $222^{\mathrm{ns}}$ & $151^{\mathrm{ns}}$ \\
\hline Cultivares (C) & 3 & $0,03 *$ & $1,6 *$ & $2,4^{*}$ & $16274 *$ & $3015^{*}$ \\
\hline Doses (D) & 3 & 2,89 & 458,4 & 969,1 & 533492 & 190231 \\
\hline $\mathrm{C} \times \mathrm{D}$ & 9 & $0,03 *$ & $2,5^{*}$ & $3,2 *$ & $3291^{*}$ & $1535^{*}$ \\
\hline Doses/ Brasil 48 & 3 & 0,80 & 136,4 & 272,9 & 123775 & 55932 \\
\hline Linear & 1 & $2,24+$ & $378,2+$ & $766,6+$ & $353381+$ & $151623+$ \\
\hline Quadrático & 1 & $0,12+$ & $16,7+$ & $43,0+$ & $4658+$ & $10983+$ \\
\hline Cúbico & 1 & $0,05^{\mathrm{ns}}$ & $14,2+$ & $8,9+$ & $13287+$ & $5190+$ \\
\hline Doses/Grand Rapids & 3 & 0,72 & 118,9 & 270,9 & 175519 & 56289 \\
\hline Linear & 1 & $2,03+$ & $321,2+$ & $761,4+$ & $437784+$ & $153440+$ \\
\hline Quadrático & 1 & $0,05+$ & $19,2+$ & $38,8+$ & $729^{\mathrm{ns}}$ & $7788+$ \\
\hline Cúbico & 1 & $0,07+$ & $16,4+$ & $12,6+$ & $88046+$ & $7640+$ \\
\hline Doses/Vitória V. Clara & 3 & 0,60 & 99,2 & 201,1 & 121459 & 44599 \\
\hline Linear & 1 & $1,78+$ & $296,2+$ & $587,6+$ & $307272+$ & $126978+$ \\
\hline Quadrático & 1 & $0,01^{\mathrm{ns}}$ & $1,5^{\mathrm{ns}}$ & $13,5+$ & $182^{\mathrm{ns}}$ & $5446+$ \\
\hline Cúbico & 1 & $0,01^{\mathrm{ns}}$ & $0,07^{\mathrm{ns}}$ & $2,3+$ & $56927+$ & $1374+$ \\
\hline Doses/Regina 71 & 3 & 0,86 & 111,2 & 233,6 & 122612 & 38016 \\
\hline Linear & 1 & $2,35+$ & $326,1+$ & $672,0+$ & $305786+$ & $113085+$ \\
\hline Quadrático & 1 & $0,07+$ & $6,5+$ & $27,4+$ & $0,25^{\mathrm{ns}}$ & $344^{\mathrm{ns}}$ \\
\hline Cúbico & 1 & $0,16+$ & $1,1^{\mathrm{ns}}$ & $1,5^{\mathrm{ns}}$ & $62049+$ & $619+$ \\
\hline Resíduo & 45 & 0,01 & 1,1 & 0,4 & 514 & 116 \\
\hline Média & & 2,66 & 6,9 & 10,8 & 200 & 146 \\
\hline C.V. $(\%)$ & & 4,00 & 14,9 & 6,2 & 11,3 & 7,3 \\
\hline
\end{tabular}

* Efeito significativo pelo teste de F em $5 \%$ de probabilidade para cultivares e interação; ns: não significativo.

+ Efeito linear e/ou quadrático e/ou cúbico significativo para doses dentro de cada cultivar, pelo teste de $\mathrm{F}$ em $5 \%$ de probabilidade de erro.

Houve efeito dos cultivares e da interação cultivar $\mathrm{x}$ dose sobre o teor de $\mathrm{Zn}$ no substrato (tabela 3). Na tabela 5 , pode-se observar o efeito quadrático das doses de composto sobre o teor de $\mathrm{Zn}$ no substrato de cada cultivar, aproximando-se dos resultados obtidos por SANTOS et al. (1998). As doses estimadas do teor máximo de $\mathrm{Zn}$ no solo foram de 63,5, 62,0, 77,8, 69,5t ha ${ }^{-1}$ para 'Brasil 48', 'Grand Rapids' 'Vitória Verde Clara'e 'Regina 71', respectivamente. COSTA (1994) verificou aumento no teor de $\mathrm{Zn}$ disponível no solo, ao adubar alface com doses crescentes de composto orgânico de lixo urbano. Resultado semelhante foi obtido por ALCOFORADO e TRINDADE (1993), ao usarem doses crescentes de composto orgânico de lixo urbano em milho.

Houve efeito de cultivares sobre o teor de $\mathrm{Cu}$ do substrato, mas não da interação cultivar $\mathrm{x}$ dose (tabela 4). O teor de $\mathrm{Cu}$ do substrato onde foi cultivado 'Grand Rapids' diferiu estatisticamente dos demais com média de $2,47 \mathrm{mg} \mathrm{dm}^{-3}$, enquanto 'Brasil 48', 'Vitória Verde Clara' e 'Regina 71' apresentam médias de $1,93,1,89$ e $1,99 \mathrm{mg} \mathrm{dm}^{-3}$, respectivamente. SANTOS et al. (1998), trabalhando com dez cultivares de alface e $35 \mathrm{t} \mathrm{ha}^{-1}$ de composto de lixo urbano, também detectaram efeito de cultivares sobre o teor de $\mathrm{Cu}$ no substrato, embora não tenha ocorrido diferença significativa entre o teor de $\mathrm{Cu}$ nos substratos de 'Brasil 48', 'Regina 71', 'Grand Rapids' e 'Vitória Verde Clara'. O efeito das doses sobre o teor de $\mathrm{Cu}$ dos substratos foi linear e crescente (tabela 5). COSTA (1994), trabalhando com o cultivar Brasil 48 em Latossolo vermelho-amarelo franco argiloso também detectou efeito linear crescente das doses $\left(0,30,60,90 \mathrm{tha}^{-1}\right)$ de composto sobre o teor de $\mathrm{Cu}$ disponível no substrato.

A análise de variância do teor de Mn do substrato revelou efeito significativo dos cultivares e não significativo da interação cultivar x dose (tabela 4). As doses elevaram o teor de Mn no substrato ajustando-se o mo delo quadrático para tal efeito(tabela 5). O teor máximo estimado de Mn no substrato foi encontrado na dose de 63,9t ha $^{-1}$. O substratos de 'Brasil 48' apresentou o valor de 9,59 $\mathrm{mg} \mathrm{dm}^{-3}$ de $\mathrm{Mn}$, enquanto 'Grand Rapids', 'Vitória Verde Clara' e 'Regina 71' apresentaram $9,29,8,86$ e $8,56 \mathrm{mg} \mathrm{dm}^{-3}$, respectivamente. Isso mostra que 'Brasil 48' absorveu menos e 'Regina 71' absorveu mais Mn. GENEVINI et al. (1991) também verificaram aumento no teor de Mn no solo em função de doses de composto orgânico de lixo urbano, que variou de $6,07 \mathrm{mg} \cdot \mathrm{dm}^{-3}$ (sem composto) a $7,76 \mathrm{mg} \mathrm{dm}^{-3}\left(42 \mathrm{tha}^{-1}\right)$.

Houve efeito dos cultivares e da interação cultivar x dose sobre o teor de $\mathrm{K}$ do substrato (tabela 3). O efeito das doses foi quadrático no substrato de 'Brasil 48' e linear nos demais (tabela 5). A dose estimada do teor máximo de $\mathrm{K}$, no solo, foi de $93,9 \mathrm{t}$ ha $^{-1}$ para 'Brasil 48'. No trabalho desenvolvido por SANTOS et al. (1998), com dez cultivares de alface e $35 \mathrm{t} \mathrm{ha}^{-1}$ de composto de lixo, os substratos onde 
Tabela 4 - Resumo da análise de variância dos valores do pH em água, da condutividade elétrica (CE) e dos teores de $\mathrm{Cu}$ e $\mathrm{Mn}$ dos substratos após colheita dos cultivares de alface adubadas com composto orgânico de lixo urbano, UFV, Viçosa, MG. 1993.

\begin{tabular}{|c|c|c|c|c|c|}
\hline \multirow[b]{2}{*}{ F.V. } & \multirow[b]{2}{*}{ G.L. } & \multicolumn{4}{|c|}{ Quadrados médios } \\
\hline & & $\mathrm{Cu}$ & $\mathrm{Mn}$ & $\mathrm{CE}$ & $\mathrm{pH}$ \\
\hline Blocos & 3 & $0,88^{\mathrm{ns}}$ & $0,36^{\mathrm{ns}}$ & $0,15^{\mathrm{ns}}$ & $0,03^{\mathrm{ns}}$ \\
\hline Cultivares (C) & 3 & $1,15 *$ & $3,29 *$ & $0,71^{\mathrm{ns}}$ & $0,05^{\mathrm{ns}}$ \\
\hline Doses (D) & 3 & 32,87 & 175,97 & 54,13 & 9,41 \\
\hline Linear & 1 & $98,31+$ & $505,71+$ & $152,67+$ & $26,62+$ \\
\hline Quadrático & 1 & $0,27^{\mathrm{ns}}$ & $21,52+$ & $1,01^{\mathrm{ns}}$ & $1,47+$ \\
\hline Cúbico & 1 & $0,02^{\mathrm{ns}}$ & $0,68^{\mathrm{ns}}$ & $8,71+$ & $0,15+$ \\
\hline $\mathrm{C} \times \mathrm{D}$ & 9 & $0,73^{\text {ns }}$ & $0,60^{\mathrm{ns}}$ & $0,54^{\mathrm{ns}}$ & $0,02^{\mathrm{ns}}$ \\
\hline Resíduo & 45 & 0,37 & 0,41 & 0,28 & 0,02 \\
\hline Média & & 2,07 & 9,07 & 2,98 & 5,98 \\
\hline C.V. (\%) & & 29,19 & 7,09 & 17,65 & 2,22 \\
\hline
\end{tabular}

* Efeito significativo pelo teste de $\mathrm{F}$ em $5 \%$ de probabilidade para cultivares e interação; ns: não significativo.

+ Efeito linear e/ou quadrático e/ou cúbico significativo para doses, pelo teste de $\mathrm{F}$ em $5 \%$ de probabilidade de erro.

foram cultivados 'Brasil 48' e 'Grand Rapids' também apresentaram altos teores de $\mathrm{K}$ e os substratos onde foram cultivados 'Vitória Verde Clara' e 'Regina71', baixos teores de $K$ no substrato. ALCOFORADO e TRINDADE (1993) também constataram aumento no teor de $\mathrm{K}$ com o aumento das doses, após incubação do solo com composto orgânico de lixo. FRITZ e VENTER (1988) e CABRERA et al. (1989) também observaram aumento no teor de metais pesados e $\mathrm{K}$ no solo com o uso de composto orgânico de lixo urbano.

A análise de variância do teor de $\mathrm{Na}$ no substrato revelou efeito significativo dos cultivares e da interação cultivar x dose (tabela 3). ). O efeito das doses foi quadrático para 'Brasil 48', 'Grand Rapids' e 'Vitória Verde Clara' e linear para 'Regina 71' (tabela 5). As doses estimadas do teor máximo de $\mathrm{Na}$, no solo, foram de 55,0, 61,9 e 64,3t ha ${ }^{-1}$ para 'Brasil 48', 'Grand Rapids' e 'Vitória Verde Clara', respectivamente. SANTOS et al. (1998) observaram que os teores de Na nos substratos de 'Brasil 48' e 'Grand Rapids', que receberam $35 \mathrm{tha}^{-1}$ de composto, foram também superiores aos de 'Vitória Verde Clara' e 'Regina 71'. No trabalho de COSTA (1994), o teor de $\mathrm{Na}$ disponível no substrato alcançou níveis elevados, em virtude do alto teor deste elemento no composto orgânico de lixo $\left(1091,9 \mathrm{mg} \mathrm{dm}^{-3}\right)$; foi estimado que o teor de Na na testemunha, após colheita de 'Brasil 48', foi $0,155 \mathrm{mg} \mathrm{dm}^{-3}$ e alcançou 191,37 e $353,25 \mathrm{mg} \mathrm{dm}^{-3}$ quando o tratamento foi de 30 e $60 \mathrm{t}$ $\mathrm{ha}^{-1}$ de composto orgânico de lixo urbano, respectivamente.

A CE no extrato de saturação do substrato, após o cultivo de alface, foi influenciada apenas pelas doses de composto (tabela 4), contrastando com resultado obtido por SANTOS et al. (1998), no qual os cultivares influenciaram significativamente a CE. Com a elevação das doses, ocorreu a elevação da CE, ajustando-se o modelo linear (tabela 5), que deve ser aceito com restrição, pois o desvio foi significativo. $\mathrm{Na}$ maior dose, antes do plantio, a CE no extrato de saturação do substrato alcançou, em média, $5,9 \mathrm{dS} \mathrm{m}^{-1}$ (tabela 2), valor superior a $4,0 \mathrm{dS} \mathrm{m} \mathrm{m}^{-1}$, que caracteriza um solo salino (BUCKMAN e BRADY, 1979). A elevação da CE do extrato de saturação do solo é comum após a aplicação de composto orgânico de lixo urbano ao solo devido aos altos teores de $\mathrm{K}$ e $\mathrm{Na}$ desses materiais (PETRUZZELLI $\boldsymbol{e t}$ al., 1989 e GENEVINI $\boldsymbol{e}$ t al., 1991). HERNÁNDEZ et al. (1992) observaram que a $\mathrm{CE}$, inicialmente $2,86 \mathrm{dS} \mathrm{\textrm {m } ^ { - 1 }}$, alcançou $4,28 \mathrm{dS} \mathrm{m}^{-1}$ quando adicionaram ao solo $180 \mathrm{t} \mathrm{ha}^{-1}$ de composto orgânico de lixo urbano. COSTA (1994) também constatou aumento da CE do extrato de saturação dos substratos, com a elevação das doses de composto de lixo em cultivo de alface, cultivar Brasil 48, em Latossolo vermelho-amarelo; sem composto, a CE média foi $1,71 \mathrm{dS} \mathrm{m}^{-1}$ e nas doses 30 e $60 \mathrm{t} \mathrm{ha}^{-1}$ a CE média foi 3,34 e 10,29 $\mathrm{dS} . \mathrm{m}^{-1}$, respectivamente. Se fossem usadas $52,5 \mathrm{tha}^{-1}$ do composto utilizado por COSTA (1994) ter-se-ia uma CE estimada de $9,0 \mathrm{dS} \mathrm{m}{ }^{-1}$, enquanto $52,5 \mathrm{tha}^{-1}$ do composto utilizado neste trabalho elevou a $\mathrm{CE}$ a $6,67 \mathrm{dS} \mathrm{m}^{-1}$, média dos quatro cultivares. Ambos os compostos são oriundos do Rio de Janeiro-RJ, porém de usinas e épocas diferentes, o que mostra a variabilidade das características de tais materiais. $\mathrm{O}$ autor concluiu que o aumento da concentração salina foi um dos principais efeitos do uso do composto orgânico de lixo.

Somente as doses de composto tiveram efeito sobre o $\mathrm{pH}$ do substrato (tabela 4), ajustandose o modelo quadrático que deve ser aceito com restrição, pois o desvio da regressão foi significativo (tabelas 4 e 5). A elevação do pH dos substratos com as doses de composto orgânico de lixo urbano pode ser observada na tabela 5 . O aumento do $\mathrm{pH}$ com as doses de composto é uma resposta comum nos solos (GENEVINI et al., 1991; ALCOFORADO \& TRINDADE, 1993; HERNANDO et al., 1989; HERNÁNDEZ et al., 1992 e COSTA, 1994). Mazur (1983), citado por KIEHL (1985), constatou a elevação do $\mathrm{pH}$ de 5,2 para 5,7 quando empregou composto de lixo urbano para determinar a influência da 
Tabela 5 - Equações ajustadas dos teores de $\mathrm{Pb}, \mathrm{Zn}, \mathrm{Mn}, \mathrm{Cu}, \mathrm{K}$ e $\mathrm{Na}$ e dos valores de $\mathrm{pH}$, condutividade elétrica (CE) e carbono orgânico (CO) de acordo com as doses $\left(\mathrm{X}=0\right.$ até $\left.52,5 \mathrm{t} \mathrm{ha}^{-1}\right)$ de composto orgânico de lixo urbano e cultivares, pontos de máxima e coeficiente de determinação $\left(\mathrm{R}^{2}\right)$, UFV, Viçosa, MG. 1993.

\begin{tabular}{|c|c|c|c|}
\hline Elemento / cultivar & Equação & $\mathrm{X} / \mathrm{Y}_{\text {máx }}$ & $\mathrm{R}^{2}$ \\
\hline \multicolumn{4}{|l|}{$\mathrm{CO} /$} \\
\hline Brasil 48 & $Y=2,099+0,034 X-0,0003 X^{2}$ & 56,7 & 96,91 \\
\hline Grand Rapids & $Y=2,140+0,028 X-0,0002 X^{2}$ & 70,0 & 95,48 \\
\hline Vitória Verde Clara & $\mathrm{Y}=2,145+0,017 \mathrm{X}$ & 52,5 & 98,97 \\
\hline Regina 71 & $Y=2,106+0,031 X-0,0002 X^{2}$ & 77,5 & 99,46 \\
\hline \multicolumn{4}{|l|}{$\mathrm{Pb} /$} \\
\hline Brasil 48 & $Y=0,147+0,424 X-0,003 X^{2}$ & 70,7 & 96,52 \\
\hline Grand Rapids & $Y=0,316+0,417 X-0,003 X^{2}$ & 69,5 & 95,39 \\
\hline Vitória Verde Clara & $\mathrm{Y}=1,050+0,220 \mathrm{X}$ & 52,5 & 99,98 \\
\hline Regina 71 & $\mathrm{Y}=0,021+0,340 \mathrm{X}-0,002 \mathrm{X}^{2}$ & 85,0 & 99,67 \\
\hline \multicolumn{4}{|c|}{ 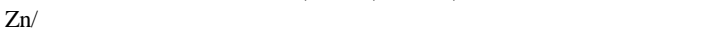 } \\
\hline Brasil 48 & $Y=0,146+0,635 X-0,005 X^{2}$ & 63,5 & 98,90 \\
\hline Grand Rapids & $Y=0,389+0,620 X-0,005 X^{2}$ & 62,0 & 98,45 \\
\hline Vitória Verde Clara & $Y=1,320+0,467 X-0,003 X^{2}$ & 77,8 & 99,61 \\
\hline Regina 71 & $Y=0,640+0,556 X-0,004 X^{2}$ & 69,5 & 99,79 \\
\hline $\mathrm{Cu}$ & $\mathrm{Y}=0,408+0,063 \mathrm{X}$ & 52,5 & 99,70 \\
\hline $\mathrm{Mn}$ & $Y=4,723+0,243 X-0,0019 X^{2}$ & 52,5 & 97,07 \\
\hline \multicolumn{4}{|l|}{$\mathrm{K} /$} \\
\hline Brasil 48 & $Y=18,113+10,521 X-0,056 X^{2}$ & 93,9 & 96,42 \\
\hline Grand Rapids & $\mathrm{Y}=1,175+8,454 \mathrm{X}$ & 52,5 & 83,28 \\
\hline Vitória Verde Clara & $Y=13,800+7,083 X$ & 52,5 & 84,38 \\
\hline Regina 71 & $Y=10,975+7,066 X$ & 52,5 & 83,13 \\
\hline \multicolumn{4}{|l|}{$\mathrm{Na} /$} \\
\hline Brasil 48 & $Y=1,745+9,467 X-0,086 X^{2}$ & 55,0 & 96,42 \\
\hline Grand Rapids & $\mathrm{Y}=3,903+8,787 \mathrm{X}-0,072 \mathrm{X}^{2}$ & 61,9 & 83,28 \\
\hline Vitória Verde Clara & $Y=4,655+7,716 X-0,060 X^{2}$ & 64,3 & 84,38 \\
\hline Regina 71 & $\mathrm{Y}=16,720+4,297 \mathrm{X}$ & 52,5 & 83,13 \\
\hline $\mathrm{CE}$ & $Y=0,908+0,079 X$ & 52,5 & 94,02 \\
\hline $\mathrm{PH}$ & $Y=4,966+0,059 X-0,0005 X^{2}$ & 52,5 & 99,47 \\
\hline
\end{tabular}

matéria orgânica no pH de um Latossolo amarelo. Segundo PETRUZZELLI et al. (1989), a aplicação de $30 \mathrm{tha}^{-1}$. ano ${ }^{-1}$ de composto orgânico de lixo elevou o pH em 0,5 unidade. COSTA (1994), cultivando alface em Latossolo vermelho-amarelo, também constatou aumentos significativos no $\mathrm{pH}(4,48 ; 7,05$; 7,95 e 8,53) com a elevação das doses de composto orgânico de lixo $\left(0,30,60\right.$ e $90 \mathrm{tha}^{-1}$, respectivamente). Sabe-se que, pelo menos temporariamente, a tendência é de que o pH se eleve com a adição de composto orgânico ao solo, principalmente por causa das bases trocáveis como $\mathrm{Ca}^{2+}, \mathrm{Mg}^{2+}, \mathrm{K}^{+}$e Na${ }^{+}$ (KIEHL, 1985), que normalmente são encontradas em alta concentração nesses materiais. A remoção dessas bases pelas plantas ou pela lixiviação é que provoca a acidificação do solo. Como nesse trabalho a lixiviação foi evitada, provavelmente, foi a remoção de bases pelas plantas que provocou uma diminuição do pH original do substrato.

\section{CONCLUSÃO}

As doses de composto orgânico de lixo urbano alteram os valores de $\mathrm{CO}, \mathrm{CE}, \mathrm{pH}$ e os teores disponíveis de $\mathrm{Pb}, \mathrm{Zn}, \mathrm{Cu}, \mathrm{Mn}, \mathrm{K}$ e Na do substrato após o cultivo da alface.

Os cultivares de alface influenciam os valores de $\mathrm{CO}$ e os teores de $\mathrm{Zn}, \mathrm{Cu}, \mathrm{Mn}, \mathrm{K}$ e Na do substrato após o cultivo.

Quanto maior as doses utilizadas de composto de lixo urbano, maior o teor de $\mathrm{Cu}, \mathrm{Mn}, \mathrm{Pb}$, $\mathrm{Zn}, \mathrm{K}$, Na e maiores o $\mathrm{CO}$, a $\mathrm{CE}$ e o $\mathrm{pH}$ do substrato após o cultivo da alface.

\section{REFERÊNCIAS BIBLIOGRÁFICAS}

ALCOFORADO, P.A.U.G., TRINDADE, A.V. Efeito do composto de lixo urbano nos teores de metais e outras características químicas do solo. Goiânia, GO, 1993. In: FREITAS, P.L. Ed. XXIV Congresso Brasileiro de Ciência do Solo, 1993. Goiânia, GO. Anais ... Goiânia, 1993, p. 307308.

AMARAL SOBRINHO, N.M.B. Interação dos metais pesados de resíduos siderúrgicos com um solo podzólico vermelhoamarelo. Viçosa, MG. 162 p. Tese (Mestrado em Fitotecnia) Curso de Pós-graduação em Fitotecnia, Universidade Federal de Viçosa, 1992.

BUCKMAN, H.O., BRADY, N.C. Natureza e propriedades dos solos. 5. ed. Rio de Janeiro: Freitas Bastos, 1979, 647 p.

CABRERA, F., DIAZ, E., MADRID, L. Effect of using urban compost as manure on soil contents of some nutrients and heavy metals. Journal Food Agricultural, v. 47, n. 1, p. 159$169,1989$.

CHANG, A.C., PAGE, A.L., WARNEKE, J.E., et al. Sequential extraction of soil heavy metals following a sludge application. Journal Environmental Quality, v. 13, n. 1, p. 33-38, 1984.

CHANYASAK, V., KATAYAMA, A., HIRAI, M.F., $\boldsymbol{e} \boldsymbol{t} \boldsymbol{a l}$. Effects of compost maturity on growth of komatsuna (Brassica rapa var. pervidis) in Neubauers's pot. I. Comparison of growth in compost treatment with that in inorganic nutrient treatments as controls. Soil Science Plant Nutrition, v. 29, n. 3, p. 239-250, 1983.

COMISSÃO DE FERTILIDADE DO SOLO DO ESTADO DE MINAS GERAIS. Recomendações para o uso de fertilizantes e corretivos em Minas Gerais; 4. Aproximação. Lavras, MG., 1989. 176 p.

COSTA, C.A. Crescimento e teor de metais pesados em alface (Lactuca sativa L.) e cenoura (Daucus carota $\mathbf{L}$.) adubadas com composto orgânico de lixo urbano. Viçosa: MG, 1994. 95 p. Dissertação (Mestrado em Fitotecnia) - Universidade Federal de Viçosa, 1994.

DEFELIPO, B.V. RIBEIRO, A.C. Análise química do solo (metodologia). Viçosa - MG: UFV, Impr. Univ., 1981. 17 p. Boletim de Extensão, 29.

DUGGAM, J.C., WILES, C. Effect of municipal compost and nitrogen fertilizer on selected soils and plants. Compendium Science, v. 17, n. 5, p. 24-31, 1976. 
FRITZ, D., VENTER, F. Heavy metals in some vegetable crops as influenced by municipal waste composts. Acta Horticutural, v. 222, n.1, p. 51-62, 1989.

GENEVINI, P.L., TANO, F., BOCCHI, S., et al. Effecto di due matrici organiche sulla fertilitá chimica del terreno e sulla disponibilitá dei metalli pesanti. Agrochemical, v. 35, p. 190198, 1991.

HE, X., TRAINA, S.J., LOGAN, T.J. Chemical properties of municipal solid waste composts. Journal Environmental Quality, v. 21, n. 3, p. 318-329, 1992.

HERNÁNDEZ, T., GARCÍA, C., COSTA, F., et al. Utilización de resíduos urbanos como fertilizantes orgánicos. Suelo y Plant, v. 2, p. 373-383, 1992.

HERNANDO, S., LOBO, M.C., POLO, A. Effect of the application of a municipal refuse compost on the physical and chemical properties of a soil. The Science of the Total Environment, v. 81, p. 589-596, 1989.

KAUSS, P. Adubo feito de lixo é tóxico. Jornal do Brasil, Rio de Janeiro, 16 dez. 1989. Cad. Cidade.

KIEHL, E.J. Fertilizantes Orgânicos. São Paulo: Ceres, 1985. $492 \mathrm{p}$.

MAEDA, S., MIZOGUCHI, M., OHKI, A., et al. Bioacumulation of zine and cadmium in freshwater alga, Chlorella vulgaris. Part I. Toxicity and accumulation. Chemosphere, v. 21, p. 953-963, 1990.

MARTINEZ, H.E.P. O cultivo hidropônico de alface (Lactuca sativa L.). In: CASALI, V.W.D. (coord.). Seminários de Olericultura. Viçosa: Universidade Federal de Viçosa, Impr. Univ., 1988. v. XV p. 74-111.

NICKLOW, C.W., COMAS-HAEZEBROUCK, P.H., FEPER, W.A. Influence of varying soil lead levels on lead uptake of leafy and root vegetables. Journal American Society Horticultural, v. 108, n. 2, p. 193-195, 1983.

PETRUZZELLI, G. Recycling wastes in agriculture: heavy metal bioavailability. Agriculture, Ecosystem and Environment, v. 27 , p. $493-503,1989$.

RHOADES, J.D. Soluble salts. In: PAGE, A.L. et al. Methods of soil analysis. Part 2 - Chemical and microbiological properties. 2. ed. Madison: American Society Agronomy, 1982. p. 167-179.

RODRIGUES, E.T. Seleção de cultivares de alface (Lactuca sativa L.) para o cultivo com composto orgânico. Viçosa: MG, 1994, 164 p. Tese (Doutorado em Fitotecnia) Universidade Federal de Viçosa, 1994.

SANTOS, I.C., CASALI, V.W.D., LOURES, E.G., et al. Teor de metais pesados, potássio e sódio em solo adubado com composto orgânico de lixo urbano após cultivo de alface. Pesquisa Agropecuária Brasileira, v. 33, n. 2, p. 157-161, 1998.

ZURERA, G., ESTRADA, B., RINCON, F., et al. Lead and cadmium contamination levels in edible vegetables. Pollution Environment Contamination Toxicology, v. 38, p. 805-812, 1987.

Ciência Rural, v. 29, n. 3, 1999. 